\title{
Studies of Short-Term Pulmonary and Peripheral Vascular Responses Induced in Oophorectomized Sheep by the Infusion of a Group B Streptococcal Extract
}

\author{
VAL G. HEMMING, ${ }^{(17)}$ WILLIAM F. O'BRIEN, GERALD W. FISCHER, STEPHEN M. GOLDEN, \\ AND SHIRLEY F. NOBLE \\ Departments of Pediatrics and Obstetrics and Gynecology, Uniformed Services University of the Health Sciences \\ and the Naval Hospital Bethesda, Bethesda, Maryland, USA
}

\begin{abstract}
Summary
Short-term (0-30 min) pulmonary and systemic vascular responses of oophorectomized ewes infused intravenously with a trichloroacetic acid (TCA) extract of a type III (strain 878) group B streptococcus (GBS) were studied. TCA-878 extract induced significant pulmonary hypertension, reduction in femoral artery pressure and reduced femoral artery $\mathrm{Po}_{2}$. These responses were similar to those observed after the infusion of sublethal doses of $E$. coli endotoxin and could be prevented by priming the animal with ibuprofen or indomethacin. Ewes rechallenged $48 \mathrm{~h}$ after their initial dose of TCA-878 extract experienced pulmonary arterial pressure significantly higher than those induced by the first infusion. Similar augmented responses were not seen after rechallenge with endotoxin. Larger doses of TCA-878 extract resulted in increasingly higher pulmonary arterial pressure. We conclude that the venous infusion of TCA extracts of GBS-878 induces significant pulmonary hypertension in sheep and that this response may be mediated by prostaglandins. The vasoactive substance extracted from these streptococci could play a role in promoting the vascular instability experienced by the human neonate with early onset GBS disease.
\end{abstract}

\section{Abbreviations}

FAP, femoral artery pressure

GBS, group B streptococci

PAP, pulmonary artery pressure

PG, prostaglandins

SDS-PAGE, sodium dodecyl sulfate-polyacrylamid gel electrophoresis

TCA, trichloroacetic acid

GBS are common causes of serious infections in the human neonate $(1,8)$. Though the clinical syndromes caused by GBS are well described, little is known of how these organisms induce the attendent physiologic abnormalities. Recently, Hellerqvist, et al. (7) and Rojas et al. (13) reported the isolation of a carbohydrate "exotoxin" from the culture media in which type III GBS had been grown. Intravenous infusion of this material was found to induce pulmonary hypertension, fever, and increase pulmonary lymphatic flow in adult sheep. At the time of the Hellerqvist and Rojas reports $(7,13)$, we were preparing TCA extracts of washed, whole GBS for utilization in studies of GBS antigens. One person in our group (W.F.O.) was utilizing the oophorectomized adult ewe model of Cefalo et al. $(2,3,11)$ to examine the early phase of vascular responses in sheep after the venous infusion of $E$. coli endotoxin. Noting some striking similarities in PAP responses induced by the Rojas' "exotoxin" and $E$. coli endotoxin, we infused sterile TCA extract of whole GBS (type III, 878) into an oophorectomized ewe. The infusion resulted in a prompt and impressive rise in PAP, a reduction in FAP, and a drop in femoral artery $\mathrm{PO}_{2}$. Once our studies had excluded endotoxin contamination of whole organism extracts (TCA-878) and produced a predictable vascular response in the available ewe model, we devised studies: 1 ) to compare the vascular responses induced by TCA-878 extract with those induced by $E$. coli endotoxin; 2) to examine responses to increasing concentrations of TCA-878;3) to determine whether, as reported with endotoxin $(3,11)$, we might inhibit the ewes' vascular responses to GBS-878 with ibuprofen or indomethacin; and 4) to study the augmentation phenomenon observed when an animal is given TCA-878 extract and then rechallenged $48 \mathrm{~h}$ later with the same extract.

\section{MATERIALS AND METHODS}

GBS bacterial extracts were prepared as follows: GBS were grown overnight in 3-liter batches in Todd-Hewitt broth (Difco Laboratories, Detroit, MI), centrifuged ( $2500 \mathrm{~g}$ for $10 \mathrm{~min})$, and the supernatant discarded. The bacterial button was suspended in $200 \mathrm{ml}$ of $2 \% \mathrm{TCA}$ and stirred overnight at $4^{\circ} \mathrm{C}$. The cell button was removed by centrifugation $(2500 \mathrm{~g}$ for $10 \mathrm{~min}$ ), the supernatant removed, and 4 volumes of absolute ethanol added. The mixture was stored overnight at $4^{\circ} \mathrm{C}$, the fine precipitate removed by centrifugation and lyophilized (The Virtis Company, Model 6212-0010, Gardiner, NY).

TCA-878 was subjected to carbohydrate analysis in the laboratory of Dr. Barry Gray utilizing the methods reported by Pritchard et al. (12). SDS-PAGE was performed on a vertical gel electrophoresis system (BRL, Model V16, Rockville, MD) utilizing standard methods. Protein concentrations of the extracts were determined utilizing the Bio-Rad protein assay (Bio-Rad Laboratories, Richmond, CA) and read on a Gilford 250 spectrophotometer (Gilford Instruments Inc., Oberlin, OH). Extracts tested were for group B type and group carbohydrate antigens utilizing a modified capillary precipitin technique (9).

Studies to determine whether the TCA extracts of GBS contained or were contaminated with endotoxin included: 1) quantitative Limulus amebocyte lysate and rabbit pyrogen assays (kindly performed by the laboratory of Dr. Donald H. Hochstein of the National Bureau of Biologics); 2) utilizing the method of Selye and associates (14) whereby adult Wistar rats received slow intravenous injections of $2 \mathrm{mg}$ of lead acetate followed by either 
E. coli endotoxin $(0.3 \mathrm{mg} / \mathrm{kg})$, TCA-878 $(0.5 \mathrm{mg} / \mathrm{kg})$, or an equivalent volume of sterile non-pyrogenic physiologic saline; and 3 ) the rat model as described by Latour and Leger-Gauthier (10) was utilized to determine whether TCA-878 could, like endotoxin, induce the systemic Schwartzman reaction in the adult rat.

We used the oophorectomized adult sheep model as described by Cefalo et al. $(2,3,11)$. Adult female Dorset sheep weighing approximately $50 \mathrm{~kg}$ were oophorectomized and allowed to recover for 2 wk. Before the placement of various catheters, each ewe was anesthetized with ketamine (Parke, Davis and Company, Detroit MI), $0.2 \mathrm{mg} \cdot \mathrm{kg}^{-1} \cdot \mathrm{min}^{-1}$, placed in the supine position and allowed to breath $40 \%$ oxygen through a face mask. Indwelling catheters were placed in the common jugular vein, femoral artery, and pulmonary artery (Swan-Ganz, Edwards Laboratories, Santa Ana, CA). Blood pressure in the pulmonary and femoral arteries was continuously measured with pressure transducers (Statham P37B, Statham Company, Oxnard, CA) and recorded on a multichannel recorder (Hewlett Packard, Rockville, $M D$ ). Blood gases were determined every 5 min throughout the studies with a blood gas-analyzer (IL 813, Instrumentation Laboratories, Lexington, MA). Anesthesia, drugs, and bacterial extracts were infused as a bolus via the jugular vein catheter. Leukocyte and platelet counts were performed by standard methods.

Several groups of ewes were studied: 1 ) negative control animals which received sterile physiologic saline; 2 ) five ewes which received $E$. coli endotoxin (055:35, Difco Laboratories, Detroit, MI) at a dose of $0.3 \mathrm{mg} / \mathrm{kg} ; 3$ ) seven ewes which received intravenously TCA- 878 extracts in sterile physiologic saline $(0.5$ $\mathrm{mg} / \mathrm{ml}$ ) and to a total dose of $0.5 \mathrm{mg} / \mathrm{kg}$ per animal; 4) four ewes which received alternatively $0.5,1.0,5$, or $10 \mathrm{mg} / \mathrm{kg}$ of TCA extract of GBS-878 dissolved in sterile physiologic saline; 5) six ewes which received an initial challenge $(0.5 \mathrm{mg} / \mathrm{kg})$ and then 48 $\mathrm{hr}$ later were rechallenged with the same dose of TCA-878 extract; and 6) six ewes which were primed intravenously with $10 \mathrm{mg} / \mathrm{kg}$ of ibuprofen (The Upjohn Company, Kalamazoo, MI); two were primed with $1.5 \mathrm{mg} / \mathrm{kg}$ of indomethacin (Merck, Sharp and Dohme, West Point, PA) and then each was challenged with 0.5 $\mathrm{mg} / \mathrm{kg}$ of TCA-878 (3).

Statistics were performed using a two-tailed, Student's paired $t$ test.

\section{RESULTS}

Examination of TCA-878 extracts for endotoxin. TCA-878 extracts did not induce gelation of Limulus amebocyte lysate in a test sensitive to endotoxin levels as low as $0.125 \mu \mathrm{g} / \mathrm{ml}$. Neither did these extracts induce fever when infused into rabbits. There were no deaths among the lead acetate primed rats who received TCA-878 extracts in doses of $0.5 \mathrm{mg} / \mathrm{kg}$. All 10 controls treated with lead acetate survived as did the controls receiving only endotoxin. The 10 rats receiving both lead acetate and $E$. coli endotoxin were dead $24 \mathrm{~h}$ after injection. The six TCA-878 rechallenged rats showed no evidence of the systemic Schwartzman reaction in their kidneys as did the six endotoxin rechallenged rats.

Preliminary characterization of TCA-878 extract. Lyophilized TCA- 878 extract was approximately $20 \%$ protein. SDS-PAGE yielded protein bands corresponding with molecular weight controls at $31,000,43,000,50,000,68,000$, and 130,000 daltons, respectively. Chromatographic carbohydrate fingerprints of TCA-878 were found to be identical with those reported utilizing whole GBS cells (12) (personal communication with Dr. Barry M. Gray). TCA-878, dissolved in physiologic saline, yielded capillary precipitin rings to both group and type III rabbit antisera, although the group ring was very faint.

Sheep infusion studies. Figures 1 through 3 depict the changes in PAP, FAP, and $\mathrm{PO}_{2}$ observed after the jugular vein infusions of saline, TCA-878 extract, or E. coli endotoxin. Each animal receiving an initial infusion of endotoxin, or TCA-878 extract,

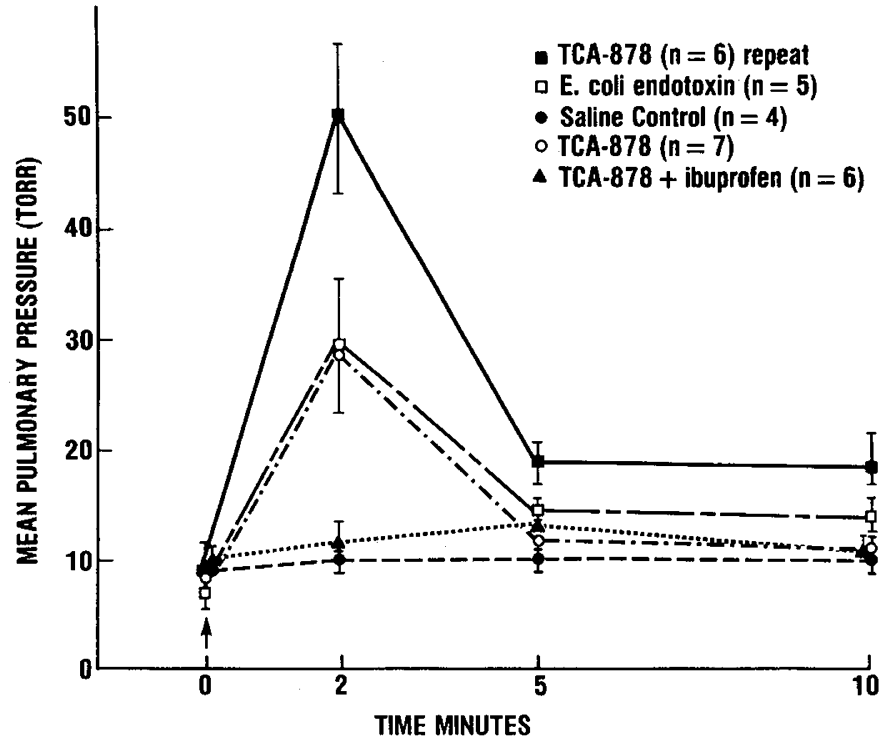

Fig. 1. Pulmonary artery pressure measured at baseline, 2, 5, and 10 $\mathrm{min}$ in oophorectomized ewes injected intravenously with $0.9 \%$ saline, extract of GBS-878, or E. coli endotoxin. Depicted are mean values \pm SE.

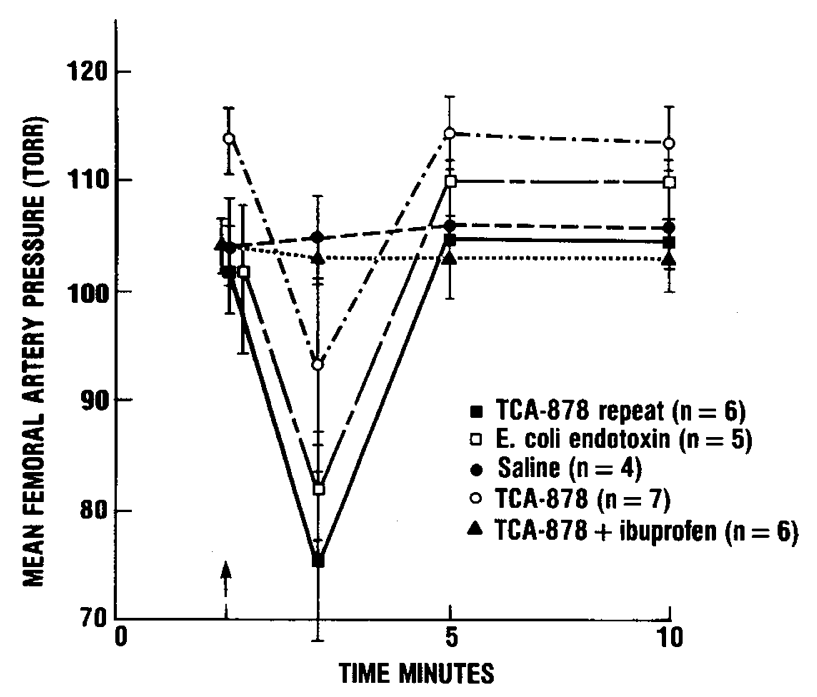

Fig. 2. Femoral arterial pressures measured at baseline, 2, 5, and 10 min in oophorectomized ewes injected intravenously with $0.9 \%$ saline, extract of GBS-878, or E. coli endotoxin. Depicted are mean values \pm SE.

demonstrated significant rises in PAP $(P<0.01)$, and decreases in FAP and $\mathrm{PO}_{2}(P<0.01$ and $P<0.05$, respectively). Six ewes, rechallenged with TCA-878 extract $48 \mathrm{~h}$ after their initial infusion, experienced a dramatic increase in PAP as compared with the initial TCA-878 response $(P<0.001)$. A commensurate reduction in $\mathrm{PO}_{2}(P<0.05)$ was also observed. Repeat exposure to $E$. coli endotoxin, performed in two sheep, failed to produce an enhanced PAP response.

As depicted in Figures 1-3, ibuprofen administered before infusion of TCA-GBS extract blocked the pulmonary and femoral arterial responses and the concurrent reduction in arterial $\mathrm{PO}_{2}$. Similar results were achieved in two animals primed with indomethacin before TCA-878 challenge.

Sheep given endotoxin (five ewes) experienced reductions in their leukocyte counts (mean $\pm \mathrm{SD}$ : baseline, $6406 \pm 1846 ; 5$ $\mathrm{min}, 1166 \pm 442$; and $10 \mathrm{~min}, 1340 \pm 947)$ and reduced platelet counts (mean $\pm \mathrm{SD}$ : baseline, $427,000 \pm 78,000 ; 5 \mathrm{~min}, 179,000$ $\pm 169,000 ; 10 \mathrm{~min} 294,000 \pm 114,000)$. In contrast, 12 ewes 


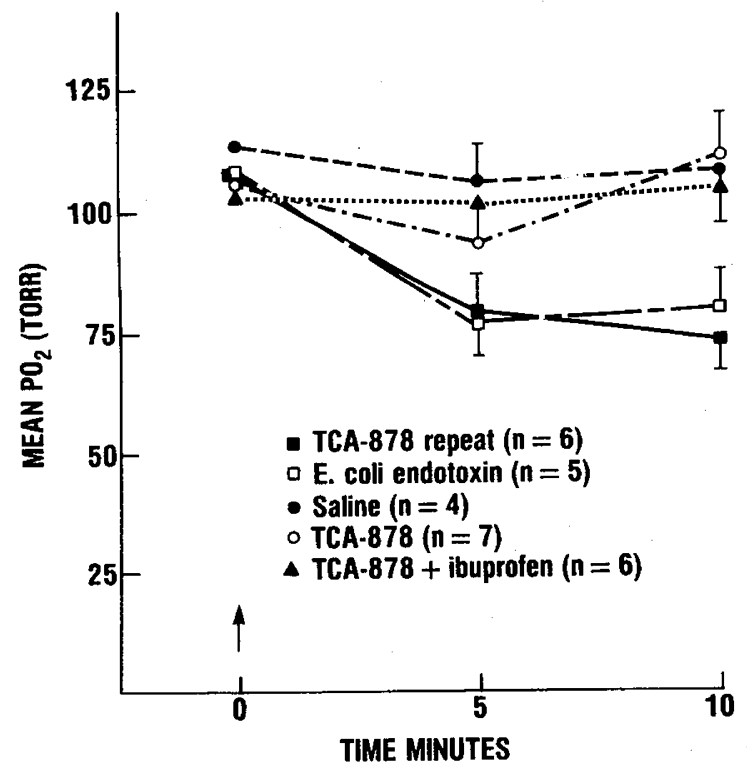

Fig. 3. $\mathrm{PO}_{2}$ measured at baseline, 5 , and $10 \mathrm{~min}$ in oophorectomized ewes injected intravenously with $0.9 \%$ saline, extract of GBS-878, or $E$. coli endotoxin. Depicted are mean values $\pm \mathrm{SE}$.

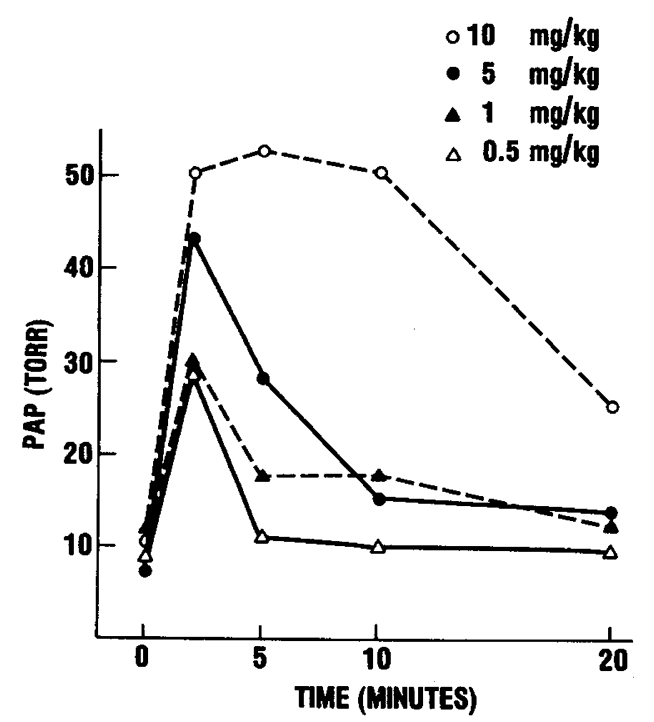

Fig. 4. Pulmonary artery pressure (PAP) measured at baseline, 2, 5, $10 \mathrm{~min}$, and $20 \mathrm{~min}$ in four oophorectomized ewes given either $0.5 \mathrm{mg} /$ $\mathrm{kg}, 1.0 \mathrm{mg} / \mathrm{kg}, 5 \mathrm{mg} / \mathrm{kg}$, or $10 \mathrm{mg} / \mathrm{kg}$ of TCA- 878 intravenously.

receiving TCA-878 experienced little change in their leukocyte counts (mean \pm SD: baseline, $6489 \pm 1412 ; 5$ min, $6455 \pm 1310$; $10 \mathrm{~min}, 6850 \pm 1329$ ) or in their platelet counts (mean $\pm \mathrm{SD}$ : baseline, $562,083 \pm 310,000 ; 5 \mathrm{~min}, 589,167 \pm 374,735 ; 10 \mathrm{~min}$, $543,750 \pm 315,271)$.

Dose response curves are portrayed in Figure 4. In separate experiments, ewes were challenged with either $0.5,1.0,5.0$, or $10.0 \mathrm{mg} / \mathrm{kg}$ of TCA-878 and their vascular responses measured. Increased doses of the extract induced higher pressures and required longer periods of time before the pressures returned to baseline levels. The changes in PAP were accompanied by reductions in FAP and arterial $\mathrm{PO}_{2}$.

\section{DISCUSSION}

Rojas et al. (13) and Hellerqvist et al. (7) recently reported recovery of an "exotoxin" from a type III GBS that induced pulmonary hypertension in adult sheep. Their "exotoxin," re- covered from the culture media but not whole bacteria, induced gelation of Limulus lysate and killed $50 \%$ of lead acetate-primed mice at concentrations of $5 \mathrm{ng} / \mathrm{ml}$ and $12 \mathrm{ng} / \mathrm{ml}$, respectively. We extracted material from whole GBS- 878 which, in addition to producing pulmonary hypertension in an endotoxin-sensitive sheep model $(2,3,11)$, also induced acute perturbations in systemic blood pressure and arterial oxygen tension. The TCA878 extract failed to induce gelation of Limulus lysate (assay sensitive to $125 \mathrm{ng} / \mathrm{ml}$ endotoxin) and did not kill lead acetateprimed rats (14). Although it is possible that TCA-878 extract and the "exotoxin" represent different substances, it is more probable that the divergent results of these assays, traditionally used to detect endotoxins, derive from differences in sensitivities of the assays utilized.

Preliminary studies demonstrated striking similarities between the vascular responses in oophorectomized ewes receiving sublethal intravenous doses of $E$. coli endotoxin and those given TCA extracts of GBS-878. Standard tests ruled out contamination of the material with endotoxin. Efforts to produce a systemic Schwartzman reaction by successive intravenous challenge of adult rats with the extract were unsuccessful as no glomerular thrombosis was observed (10). These studies suggested that despite induction of very similar vascular responses in oophorectomized ewes, there are important differences in the biologic activities of TCA-878 extract and $E$. coli endotoxin.

During studies to determine whether the GBS extract could induce a systemic Schwartzman-like response, oophorectomized ewes were infused intravenously with standard doses of endotoxin or TCA-878 and then rechallenged after $48 \mathrm{~h}$ with the same dose of the homologous material. Impressive differences in the vascular responses of the endotoxin and the TCA-878 rechallenged ewes were observed with the TCA-878 rechallenged animals experiencing the highest recorded PAP levels (Fig. 1). Augmentation of the vascular responses with TCA-878 was unexpected. We are aware of no reports of other bacteria-derived products which induce similar vascular responses in animal models. The mechanisms responsible for augmentation remain to be elucidated. Antibody-mediated or anamnestic responses seem unlikely causes because of the brief time between challenge doses. Pulmonary Schwartzman reactions appear unresponsible as we were unable to produce the enhanced response by challenge and rechallenge with $E$. coli endotoxin. Augmentation may be PG mediated as it was prevented by priming the ewes with ibuprofen before rechallenge.

Previous work, utilizing the oophorectomized ewe model demonstrated correlations between PAP in endotoxin-challenged ewes and serum levels of PGE and $F_{2 \alpha}$ (3). Sheep primed with ibuprofen or indomethacin and then challenged with endotoxin failed to develop increased PAP or significant increases in serum PG levels (3). Similar to the endotoxin model, ewes primed with ibuprofen or indomethacin and then challenged with TCA-878 also failed to experience pulmonary hypertension or decreases in FAP or arterial $\mathrm{PO}_{2}$. Ibuprofen and indomethacin reduce PG production by inhibiting PG synthetase $(4,5)$. The prevention of pulmonary hypertension and the other vascular changes induced by TCA-878 suggest that PG may play an important role in their genesis. Whether PG are released secondary to acute endothelial injury or perhaps by platelet or leukocyte aggregation is unknown. The latter cause seems less likely as we were unable to document reductions in either platelet counts or leukocyte counts in concert with the changes in pulmonary pressure.

One can only speculate as to whether these observations have relevance to the problem of GBS infection in the newborn. Harrison and associates reported in 1969 (6) that infusion of endotoxin in dogs resulted in injury to the endothelium of pulmonary vessels, reduction in pulmonary surfactant, and atelectasis. Hyaline membranes are commonly found in the lungs of infants who die with GBS pneumonia and sepsis $(1,8)$. Indeed, some of the early high mortality seen in early onset disease occurred because clinicians had not yet recognized the similari- 
ties between the respiratory distress syndrome (hyaline membrane disease) in the premature infant and GBS pneumonia and sepsis. To date, there are no reported PAP measurements in infants with GBS sepsis or pneumonia; however, because neonates are born with already thickened pulmonary arteriolar walls and pulmonary vessels very responsive to acidosis, hypoxemia, or hypercarbia, it seems possible that bacterial products from GBS might induce serious pulmonary vascular injury in infected infants. The injury would promote the release of vasoactive mediators such as PG decreasing pulmonary arterial blood flow thus exacerbating the infants' hypoxemia.

\section{REFERENCES AND NOTES}

1. Ablow, R. C., Driscoll, S. G., Effmann, E. L., Gros, I., Jolles, C. J., Uauy, R, and Washaw, J. B.: A comparison of early-onset group B neonatal infection and the respiratory distress syndrome of the newborn. N. Engl. J. Med., 294: 65 (1976).

2. Cefalo, R. C. and Crenshaw, C.: Effects of exogenous estrogen on $\mathrm{PO}_{2}$ and experimental endotoxemia in sheep. Am. J. Obstet. Gynecol. 120: 678 (1974).

3. Cefalo, R. C., Lewis, P. E., O’Brien, W. F., Fletcher, J. R., and Ramwell, P. $\mathrm{W} .:$ The role of prostaglandins in endotoxemia: Comparisons in response to the nonpregnant, maternal, and fetal models. Am J. Obstet. Gynecol., 137: $53(1980)$

4. Crook, D., Collins, A. J., Bacon P. A., and Chan, R.: Prostaglandin synthetase activity from human rheumatoid synovial microsomes. Ann. Rheum. Dis. 35: 327 (1976).

5. Ferreira, S. H. and Vane, J. R.: New aspects of the mode of action of nonsteroid anti-inflammatory drugs. Annu. Rev. Pharmacol., 14: 57 (1974)

6. Harrison, L. H., Beller, J. J., Hinshaw, L. B., Coalson, J. J., and Greenfield, L.
J.: Effects of endotoxin on pulmonary capillary permeability. Ultrastructure and surfactant. Surg. Gyn. Obstet., 129: 723 (1969).

7. Hellerqvist, C. G., Rojas, J., Green, R. S., Sell, S., Sundell, H., and Stahlman M. J.: Studies on group B beta hemolytic Streptococcus. I. Isolation and partial characterization of an extracellular toxin. Pediatr. Res., $15: 89$ (1981).

8. Hemming, V. G., McCloskey, D. W., and Hill, H. R.: Pneumonia in the neonate associated with group B streptococcal septicemia. Am. J. Dis. Child., 130: 1231 (1976).

9. Lancefield, R. C.: Two serological types of group B streptococci with related, but not identical, type specific substances. J. Exp. Med., 67: 25 (1938).

10. Latour, J-G and Leger-Gauthier, C.: Prostaglandins in the pathogenesis of the generalized Schwartzman reaction. Am. J. Obstet. Gynecol., 135:577 (1979).

11. O'Brien, W. F. Cefalo, R. C. Lewis, P. E. Fletcher, J. R and Ramwell, P. W.: The role of prostaglandins in endotoxemia and comparisons in response in the nonpregnant, maternal and fetal models. Am. J. Obstet. Gynecol., 138: 535 (1981).

12. Pritchard, D. G., Coligan, J. E., Speed, S. E., and Gray, B. M.: Carbohydrate fingerprints of streptococcal cells. J. Clin. Microbiol., 13:89 (1981).

13. Rojas, J., Green, R. S., Hellerqvist, C. G., Olegard, R., Brigham, K. L., and Stahlman, M. T.: Studies on group B beta hemolytic Streptococcus. II. Effects on pulmonary hemodynamics and vascular permeability in unanesthetized sheep. Pediatr. Res., 15:892 (1981)

14. Selye, H., Tuchweber, B., and Bertok, L.: Effect of lead acetate on the susceptibility of rats to bacterial endotoxins. J. Bacteriol., 91: 884 (1966).

15. The opinions herein are those of the authors and are not to be construed as reflecting the views of the Navy Department, the Uniformed Services University of the Health Sciences or the Department of Defense.

16. We thank Dominique M. Nau for excellent editorial assistance.

17. Requests for reprints should be addressed to: Dr. Val G. Hemming, Uniformed Services University, 4301 Jones Bridge Road, Bethesda, MD 20814.

18. Supported in part by USUHS Grants C08602, C08606 and CIP Grant 76-06886, Naval Hospital Bethesda, Bethesda, MD

19. Received for publication July 22, 1982.

20. Accepted for publication May 11, 1983.

\title{
Plasma Concentrations of Vitamin D Metabolites in Premature Infants
}

\author{
T. MARKESTAD(40), L. AKSNES, P. H. FINNE, AND D. AARSKOG \\ Department of Pediatrics, University of Bergen, 5016 Bergen, Norway
}

\section{Summary}

The plasma concentrations of 25-hydroxyvitamin D (OHD), $1,25-(\mathrm{OH})_{2} \mathrm{D}$ and $24,25-(\mathrm{OH})_{2} \mathrm{D}$ were determined in 28 healthy premature infants (median gestational age 33, range 28-36 wk; and median birth weight 1880 , range $900-2350 \mathrm{~g}$ ) during the first 5-10 wk of life, and in a reference group of 17 young adults. The infants received a vitamin D supplement of $500 \mathrm{IU} / \mathrm{d}$ and a diet low in calcium (Ca) and phophorus (P) compared with that of corresponding intrauterine accretion rates.

The median 25-OHD concentration increased from 11 (range 6-30) $\mathrm{ng} / \mathrm{ml}$ at $1 \mathrm{~d}$ to 27 (range, 15-41) $\mathrm{ng} / \mathrm{ml}$ by 5-10 wk of age $(P<0.01)$. 1,25-(OH $)_{2} \mathrm{D}$ concentrations at age $1 \mathrm{~d}$ were similar to the adult levels (median 37 , range 8-64 versus 35 , range $18-58 \mathrm{pg} / \mathrm{ml}$ ), but increased significantly within 1 wk to $48(26-156) \mathrm{pg} / \mathrm{ml}(P=0.01)$, and between 1 and $3-4$ wk of age to $104(58-203) \mathrm{pg} / \mathrm{ml}(P<0.01)$. The levels at 5-10 wk were similar to the 3-4 wk value. $24,25-(\mathrm{OH})_{2} \mathrm{D}$ concentrations were persistently low compared with the adult levels (medians 0.4 0.5 , range $<0.3-2.1$ versus 1.7 , range $0.4-2.0 \mathrm{ng} / \mathrm{ml}, P<0.01$ ). The relative concentrations, expressed as the ratio of 24,25 -
$(\mathrm{OH})_{2} \mathrm{D}$ to $25-\mathrm{OHD}$, were comparable to those of the adults at birth, but decreased significantly within 2 wk. The data demonstrate that healthy premature infants can produce high plasma levels of 1,25-(OH) $)_{2} \mathrm{D}$.

The main function of vitamin $\mathrm{D}$ is to enhance intestinal absorption of calcium (Ca) and phophorus (P) (reviews 12, 15). This effect, however, is first achieved after a series of metabolic alterations. The vitamin is first hydroxylated to 25-OHD in the liver, and then to $1,25-(\mathrm{OH})_{2} \mathrm{D}$, which is the principal metabolite with biologic activity, in the kidneys. The circulating concentration of $1,25-(\mathrm{OH})_{2} \mathrm{D}$ is regulated according to the body's need for $\mathrm{Ca}$ and $\mathrm{P}(12,15)$. Other vitamin $\mathrm{D}$ metabolites have also been isolated. $24,25-(\mathrm{OH})_{2} \mathrm{D}$ has received most attention, but its significance remains to be clarified $(12,15)$.

Rickets is not uncommon in premature infants $(7,23,31)$. Several factors are probably of pathogenetic importance $(5,7$, $10,25,30$ ), and deficient functions in all aspects of vitamin $D$ metabolism have been implicated, such as: intestinal absorption $(21,31,37)$, hepatic 25 -hydroxylation $(19,21,31)$, renal 1- 